\title{
Quantum dynamics in a kicked square billiards
}

\author{
${ }^{1}$ S. Rakhmanov, ${ }^{2}$ D. Babajanov, ${ }^{1,2}$ O. Karpova* ${ }^{3}$ F. Khoshimova \\ ${ }^{1}$ Faculty of Physics, National University of Uzbekistan, Vuzgorodok, Tashkent \\ 100174,Uzbekistan \\ ${ }^{2}$ Turin Polytechnic University in Tashkent, 17 Niyazov Str., Tashkent 100095, Uzbekistan \\ ${ }^{3}$ Navoi State Mining Institute, 27 Janubiy ko'chasi, Navoiy, Uzbekistan \\ *ola_july@mail.ru
}

PACS 73.63.Kv

DOI 10.17586/2220-8054-2015-6-2-216-223

We study kicked particle dynamics in a rectangular quantum billiard. The kicking potential is chosen as localized at the center of the billiard. The exact solution for the time-dependent Schrödinger equation for a single kicking period is derived. Using this solution, the time-dependence of the average kinetic energy and probability density as a function of spatial coordinates are computed. Different regimes for trapping of the particle in kicking area are analyzed. It is found that depending of the values of kicking parameters, the average kinetic energy can be a periodic or a monotonically growing function of time or can be suppressed. Such behavior is explained in terms of particle trapping regimes. Wave packet dynamics are also studied.

Keywords: quantum dynamics, billiard, Schrödinger equation.

Received: 2 February 2015

\section{State of the art and statement of the problem}

The study of particle dynamics in driven confined quantum systems is of practical importance for different newly emerging topics of nanoscale physics and nanotechnology. In fact, many nanoscale systems, materials and devices are subject to the influence of different external perturbations and environmental effects. The role of such effects in particle transport is relevant to the problem of tuning electronic, optic and acoustic properties of different nanoelectronic devices. Conversely, in confined quantum systems, particle dynamics also depend also on the geometry of the confinement boundaries. Due to these two facts driven confined systems have become an ideal testing ground for solving the problem of tunable particle transport in low-dimensional functional materials, such as quantum dots, wires networks etc. The most convenient tool for modeling of confined systems are so-called billiard geometries, or simply billiards. These are finite-size spatial domains with hard or soft walls providing confinement.

Earlier, billiards were the subject of extensive study in nonlinear dynamics [1] and quantum chaos theory [10]. In particular, it was found that classical particle dynamics in billiards strongly depended on the geometry of its boundaries. For instance, dynamics can be regular or chaotic depending on the shape of the billiard. In the quantum case, such a feature is on display in the energy spectrum of the system, implying that for billiards whose dynamics are integrable in the classical limit, level spacing distribution is always of the Poisson type, while for classically chaotic billiards, the distribution is Gaussian in nature [4].

Later, quantum billiards have found effective application as models for quantum dots in nanoscale physics [16]. Quantum dots are nanoscale domains in semiconducting structures of molecular systems, in which electronic motion is restricted to a finite domain by providing constant electric fields. It was found that by changing the shape and size of the 
dot to be modeled by the quantum billiard, one can manipulate electron transport in the dot. Despite the fact that both quantum dots and billiards have been extensively studied, most of the works on this topic are restricted by considering isolated systems, i.e. in the absence of external forces. However, driven quantum systems are more attractive from the viewpoint of environmental effects and external perturbation effects on particle transport. We note that classical and quantum dynamics of periodically driven systems were also the subject of extensive research in the past. It was found that for a periodically driven classical system, the average kinetic energy increased linearly with time, while for the corresponding quantum system, such growth is suppressed [1]. The latter is called a quantum localization phenomenon, which is, to some extent, an analog of the well-known Anderson localization in solid state physics.

In this paper, we study the quantum dynamics of a periodically driven particle confined in a square-shaped billiard. We derive an exact analytical solution for the timedependent Schrödinger equation which describes the dynamics of such a system. Using the obtained solution, we compute the average kinetic energy as function of time. Also, we analyze wave packet evolution in our system.

\section{Kicked square billiard}

Before starting the treatment of driven billiard, let us briefly recall the corresponding unperturbed system. The motion of a quantum particle in a square billiard is described by the following stationary Schrödinger equation:

$$
H_{0} \psi_{n m}=\epsilon_{n m} \psi_{n m}
$$

where

$$
\begin{aligned}
H_{0} & =-\frac{1}{2}\left(\frac{\partial^{2}}{\partial x^{2}}+\frac{\partial^{2}}{\partial y^{2}}\right), \\
\psi_{n m} & =\frac{2}{a} \sin \frac{n \pi x}{a} \sin \frac{m \pi y}{a},
\end{aligned}
$$

$a$ is the side of square,

$$
\epsilon_{n m}=\frac{\pi^{2}}{a^{2}}\left(n^{2}+m^{2}\right)
$$

are the eigenfunctions and eigenvalues, respectively.

The external potential is chosen in the form of delta kicks as:

$$
V(x, y, t)=\varepsilon(\cos x+\cos y) \sum_{l=0}^{\infty} \delta(t-l T) .
$$

This potential is a two dimensional generalization of the well-known delta-kicks studied earlier in the Ref. [1]. Schematically, the kicked billiard we are going to study can be represented as in Fig.1.

Particle dynamics in such a billiard are described by the following time-dependent Schrödinger equation:

$$
i \frac{\partial \Psi}{\partial t}=\left[H_{0}+V(x, y, t)\right] \Psi
$$

where

$$
\Psi=\Psi(x, y, t)
$$






FIG. 1. Profile of the external kicking potential

The wave function of the system, i.e. the solution of Eq.(6) can be expanded in terms of unperturbed billiard wave functions given by Eq.(3) as:

$$
\Psi(x, y, t)=\sum A_{n m}(t) \psi_{n m}(x, y),
$$

This allows us to find expansion coefficients explicitly and exactly:

$$
A_{n m}(t+T)=\sum_{n^{\prime}, m^{\prime}} A_{n^{\prime} m^{\prime}}(t) V_{n m n^{\prime} m^{\prime}} e^{-i \epsilon_{n^{\prime} m^{\prime}} T},
$$

where

$$
V_{n m n^{\prime} m^{\prime}}=\iint \psi_{n m}^{*}(x, y) e^{i \varepsilon(\cos x+\cos y)} \psi_{n^{\prime} m^{\prime}}(x, y) d x d y
$$

In the derivation of this expression, we used the relation:

$$
e^{i \varepsilon \cos x}=\sum_{l} i^{l} J_{l}(\varepsilon) e^{i l x}
$$

We note that the expansion coefficients obey the normalization condition:

$$
\sum_{n, m}\left|A_{n m}(0)\right|^{2}=1
$$

One important characteristic of the dynamics in driven systems is the average kinetic energy of a driven particle. For our system, it can be defined as:

$$
E(t)=\int \Psi^{*}(x, y, t) H_{0} \Psi(x, y, t) d x d y=\sum_{n, m}\left|A_{n m}(t)\right|^{2} \epsilon_{n m} .
$$




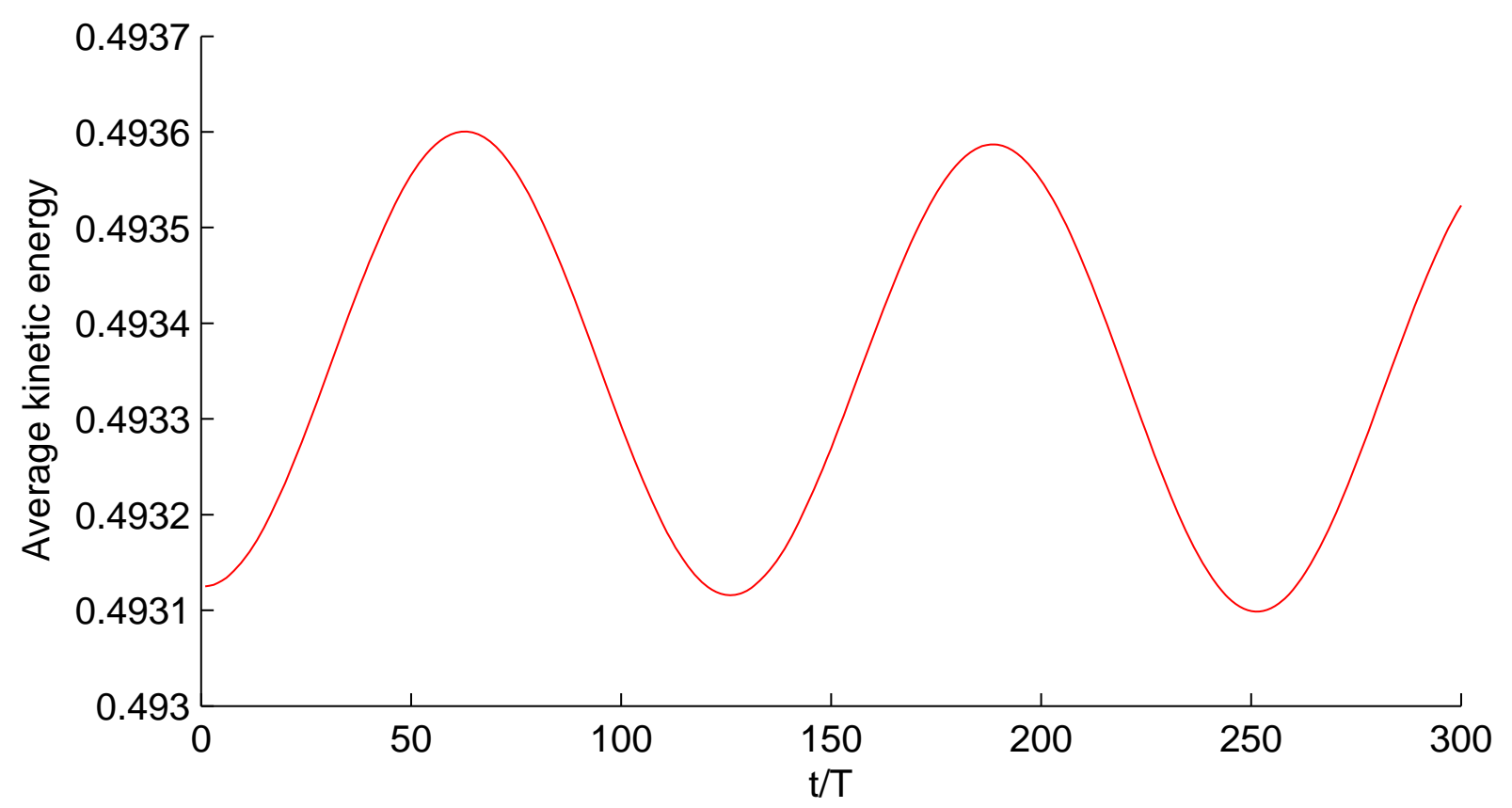

FIG. 2. Time dependence of the average kinetic energy $(\varepsilon=0.001, T=0.1)$



FIG. 3. Time dependence of the average kinetic energy for fixed $\varepsilon$ at different values of the kicking period $\left(\varepsilon=0.001, T_{1}=0.01, T_{2}=0.001\right)$

\section{Particle dynamics in a kicked square billiard}

We are interested in analyzing this quantity as a function of time. In calculating the average kinetic energy, we take a few non-zero initial values of the expansion coefficients which obey the above normalization conditions given by (11). In Fig.2, $E(t)$ is plotted.

As is seen from these plots, $E(t)$ is periodic in time with a period much higher than that of the kicking force. Figs. 3 and 4 present the average kinetic energy as a function of time for fixed $T$ at fixed kicking strength, $\varepsilon$. 


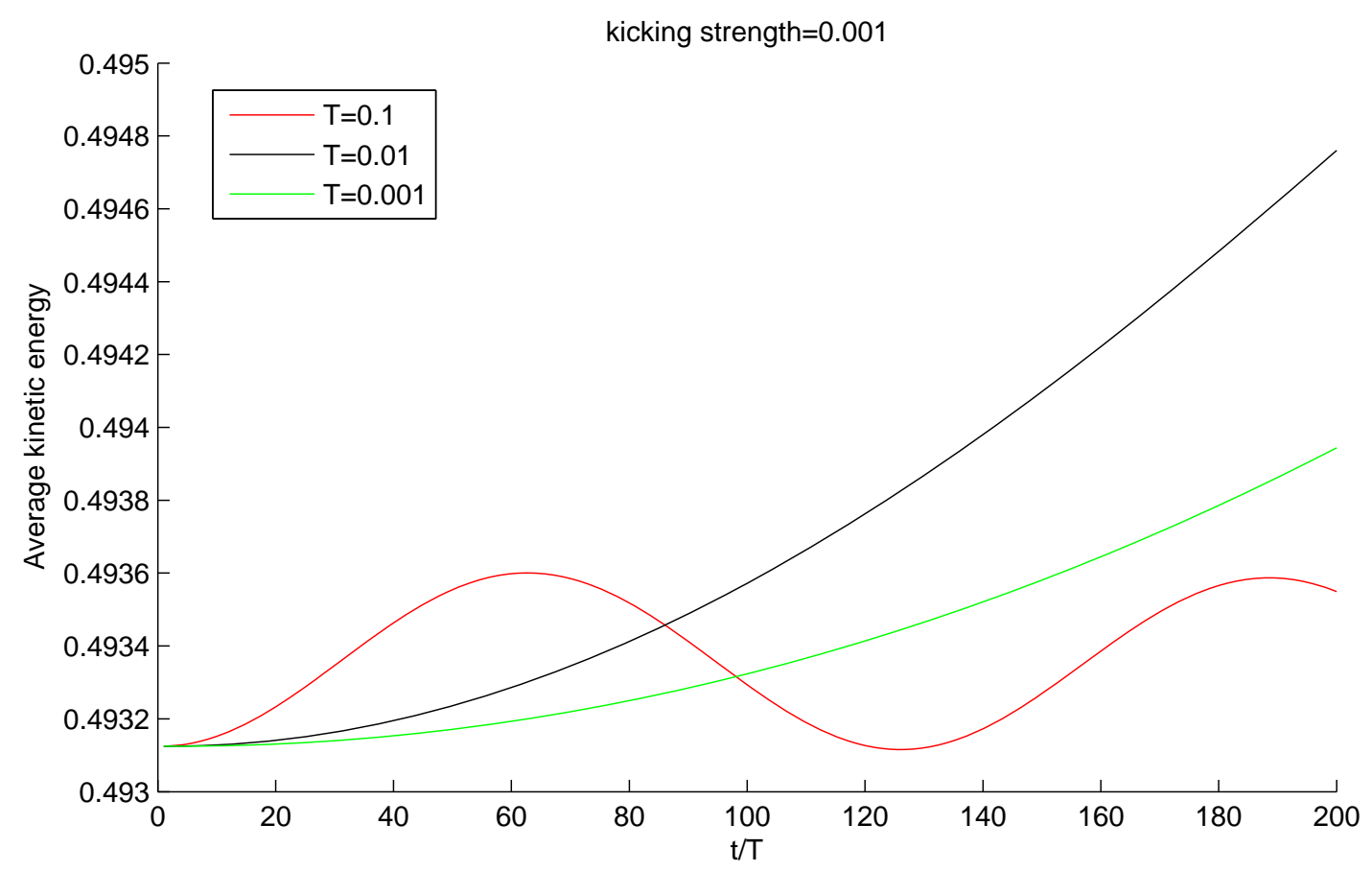

FIG. 4. Time dependence of the average kinetic energy for fixed $\varepsilon$ at different kicking period values $\left(\varepsilon=0.001, T_{1}=0.1, T_{2}=0.01, T_{3}=0.001\right)$



FIG. 5. Average kinetic energy as a function of kicking strength and period ( at $t=20 T)$

One can observe from these plots that the profile of $E(t)$ depends on the kicking period. The dependence of the average kinetic energy upon the kicking parameters can clearly be seen from the plot in Fig. 5, where it is plotted as a function of $T$ and $\varepsilon$. This plot shows that the growth of $E(t)$ is as intense as higher $\varepsilon$ and as shorter $T$. To explain the above behavior of $E(t)$, we analyzed the spatio-temporal evolution of the probability density for our system, $|\Psi(x, y, t)|^{2}$. Figs. 6 and 7 present comparison of $|\Psi(x, y, t)|^{2}$ at different moments of time with the profile of the kicking potential. It is clear from these plots that the localization 
Probability density

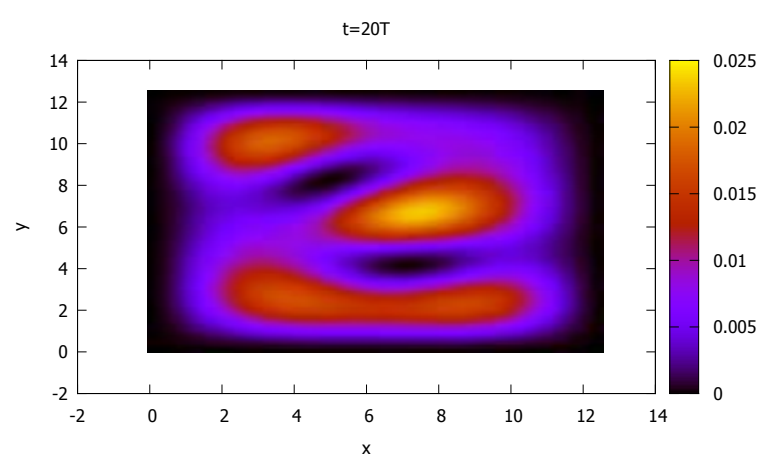

Kicking potential



FIG. 6. Probability density versus coordinates $(t=20 T, \varepsilon=0.001, T=1.26)$

Probability density

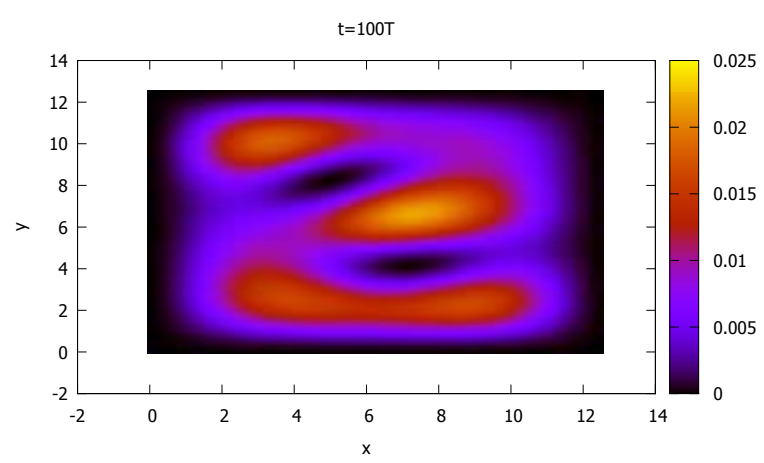

Kicking potential

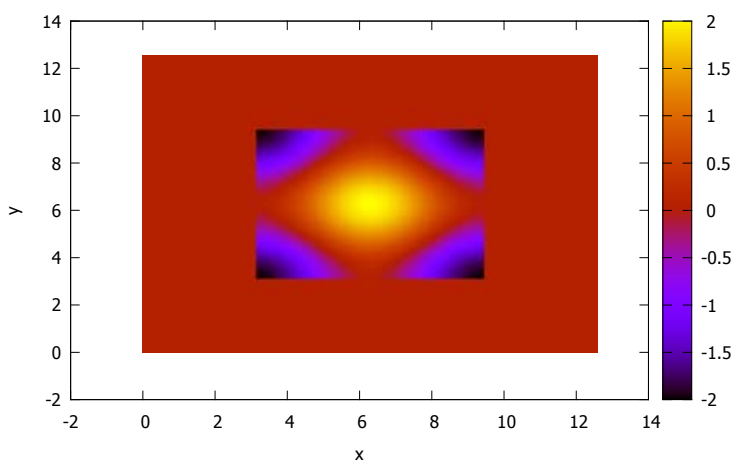

FIG. 7. Probability density versus coordinates. $(t=100 T, \varepsilon=0.001, T=1.26)$

of the probability density is periodic in time, i.e. probability density is periodically localized on the areas of billiard where the kicking potential is negative and positive. If the particle motion is localized on the area where the kicking potential is repulsive (positive), it gains the energy, while on the area where the potential is negative, it looses energy. In other words, particle is periodically trapped on the areas where it gains and loses energy. This is the reason for the time-periodic behavior of the average kinetic energy.

\section{Wave packet evolution}

Another characteristic of particle transport in driven systems is the wave packet dynamics, i.e. the evolution of the packet profile in space and time. In this work, we consider evolution of Gaussian wave packets, i.e. we assume that at $t=0$ the wave packet has a Gaussian profile as:

$$
\Psi(x, y, 0)=\Phi(x, y)=\frac{1}{\pi \sqrt{\pi}} \exp \left(-\frac{x^{2}+y^{2}}{2 d^{2}}\right) \sum_{n, m} \sin \frac{\pi n x}{a} \sin \frac{\pi m y}{a} .
$$

Then, the expansion coefficients at $t=0$ can be expressed via the wave packet as:

$$
A_{m n}(0)=\frac{1}{\pi \sqrt{\pi}} \exp \left(-\frac{x^{2}+y^{2}}{2 d^{2}}\right),
$$

where $d$ is the width of packet. 


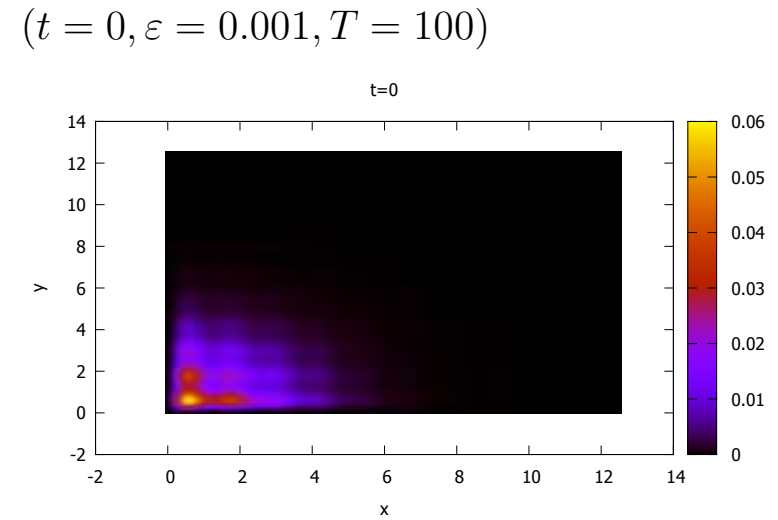

$(t=31 T, \varepsilon=0.001, T=100)$

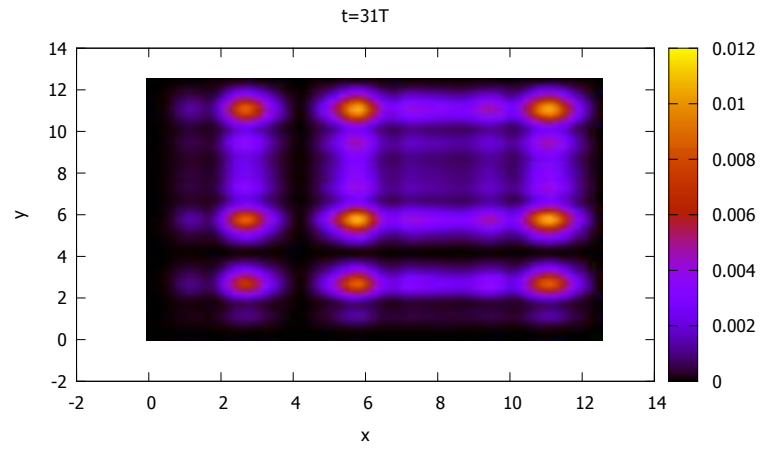

$$
(t=3 T, \varepsilon=0.001, T=100)
$$

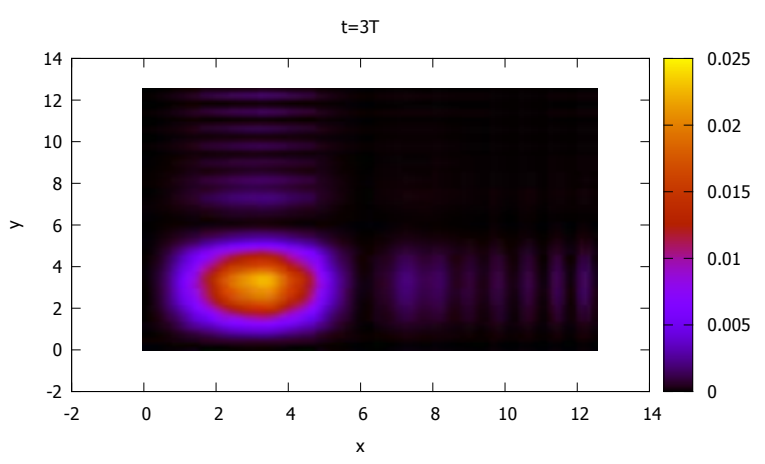

$(t=46 T, \varepsilon=0.001, T=100)$

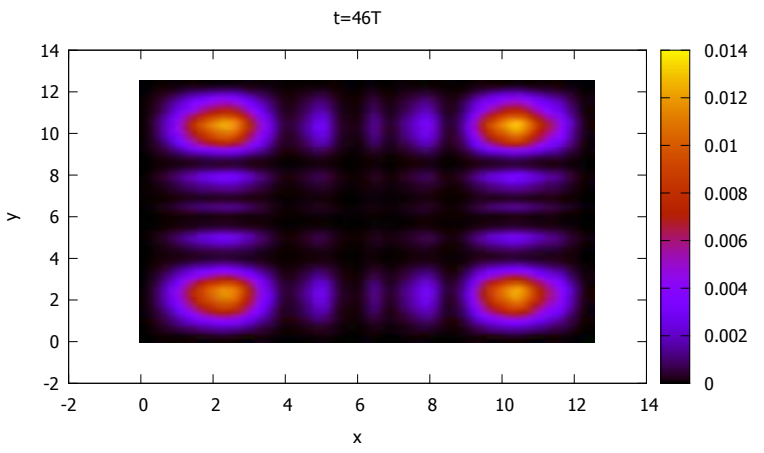

FIG. 8. Wave packet evolution

In upper left panel of Fig. 8, the time evolution of the wave packet is presented for the moment $t=0$ for the values of the kicking parameters $\varepsilon=0.001, T=100$. Other panels of Fig. 8 depict the time evolution of the wave packet for the following time points $t_{1}=3 T, t_{2}=31 T, t_{3}=46 T$ for the values of the kicking parameters $\varepsilon=0.001, T=100$. As is seen from these plots, the dispersion of the packet occurs after certain number of kicks.

\section{Conclusions}

Summarizing, in this work, we have studied the quantum dynamics of a particle confined in a square billiard and interacting with an external time-periodic force having the form of delta-kicks. The system is described by the time-dependent Schrödinger equation. An exact solution for this time-dependent Schrödinger equation is found during single kicking period. Using the obtained solution, characteristics of the particle dynamics, such as the average kinetic energy, solution of the single state energy, probability density and wave packet transport can be computed. The average kinetic energy was found to be periodic in time. This periodicity was shown to be a result of particle interaction, which is periodically located in areas where the kicking force is attractive or repulsive. When particle is confined to a negative force area, it continuously loses its energy, and conversely, when trapped on a positive force area, it gains energy. Similar behavior was found for single state energy. Analysis of the wave packet profile in time and space showed that after a certain number of kicks and collisions with the billiard wall, dispersion of the Gaussian wave packet occurs. The results obtained in this work can be useful for the problem of tunable Fermi acceleration 
in quantum systems, and tunable electronic transport in nanoscale devices, e.g. quantum dots, wires, wells etc.

\section{References}

[1] G. Casati, B.V. Chirikov, J. Ford, F.M. Izrailev, in: G. Casati, J.Ford (Eds.), Lecture Notes in Physics, vol. 93, Springer-Verlag, Berlin, p. 334 (1979).

[2] Lichtenberg A.J., Lieberman M.A. Regular and stochastic motion. N.Y.-Heidelberg-Berlin, SpringerVerlag, 499 pp. (1983).

[3] R.Z. Sagdeev, D.A. Usikov, G.M. Zaslavsky, Nonlinear Physics: From Pendulum to Turbulence and Chaos. Academic Publisher, New York (1988).

[4] M.C. Gutzwiller, Chaos in classical and quantum systems, New York, Springer Verlag (1990).

[5] Bagrov V.G. and Gitman D.M. Exact Solutions of Relativistic Wave Equations, Dordrecht, Kluwer (1990).

[6] A.J. Lichtenberg, M.A. Lieberman, Regular and Chaotic Dy-namics. Springer-Verlag, New York (1992).

[7] Thaller B. The Dirac Equation. Berlin, Springer (1992).

[8] M.F. Shlesinger, G.M. Zaslavsky, U. Frish (Eds.), Lvy Flights and Related Topics in Physics. SpringerVerlag (1995).

[9] G. Casati and B.V. Chirikov(eds.) Quantum chaos between order and disorder a selection of papers. Cambridge University Press, New York (1995).

[10] Hans-Jürgen Stöckmann. Quantum Chaos: An Introduction. Cambridge University Press (1995).

[11] De Vincenzo S. Thesis Magister scientiarum in science, physics mention. Universidad Central de Venezuela, Caracas (1996). (in Spanish)

[12] K. Nakamura Quantum Versus Chaos. Questions Emerging from Mesoscopic Cosmos. Kluwer Academic Dordrecht (1997).

[13] Yoseph Imry. Introduction to Mesoscopic Physics. Oxford University Press (1997).

[14] H.-J. Stöckmann, Quantum Chaos: An Introduction. Cambridge University Press, Cambridge, UK (1999).

[15] K. Richter, Semiclassical Theory of Mesoscopic Quantum Systems. Springer, Berlin (2000).

[16] K. Nakamura and T. Harayama. Quantum Chaos and Quantum Dots. Oxford University Press (2004). 